Universidad Autónoma del Estado de México

(1) @ 9

UחED JAEN

\section{Tecnologías emergentes en la enseñanza del cálculo diferencial para ingeniería}

Centro Asociado "Andres de Vandelvira" $\mathrm{e}^{x i} \mathrm{~d} \theta 19$

Magally Martínez Reyes Anabelem Soberanes Martín René G. Cruz Flores 


\section{Introducción}

La Tercera Revolución Industrial se caracterizó por el uso de la electrónica y la tecnología de la información, la automatización de la producción y la globalización avanzada en todos los sectores de la sociedad.

La Cuarta Revolución Industrial suma la convergencia de nuevas tecnologías incluyendo la nanotecnología, la inteligencia artificial, la robótica, la genética y la impresión 3D, lo que modifica a la sociedad y el papel del sector educativo. 


\section{Introducción}

La constante evolución de la educación superior requiere ir adaptando las estrategias y herramientas de enseñanza a las características de los estudiantes que ya para la generación actual son nativos tecnológicos, en este escenario las tecnologías de la enseñanza virtual (realidad aumentada, realidad virtual, app, simulación móvil, sistemas tutoriales inteligentes móviles, etcétera) se combina con estrategias tradicionales para dar lugar al aprendizaje combinado (blended learning).
Una pedagogía para este modelo tendría que ser el resultado de la combinación del fácil acceso a la información con el uso de tecnologías relacionadas y la enseñanza presencial, con la intención de facilitar experiencias de instrucción independientes y colaborativas. 


\section{Introducción}

El acceso a las herramientas en la web (eventos y cursos on-line, seminarios web, videos, tutoriales, wikis, etc.) y la diversificación de recursos en los dispositivos móviles (RA, RV, STI-m, app) ofrecen a los alumnos contenido para estudiar en su propio tiempo y ritmo con enfoques autodirigidos, pero bajo la premisa de ser discutidos o analizados en aula de clase.

En este contexto, la propuesta de las tecnologías emergentes es una opción para contribuir a la problemática de la enseñanza y aprendizaje del cálculo (diferencial e integral) a nivel superior en carreras de ingeniería, en particular porque los estudiantes tienen acceso a dispositivos tecnológicos y suelen tener mayor disposición para aprender el manejo de los mismos, en un principio con fines de comunicación en redes sociales y posteriormente como apoyo a su proceso educativo. 


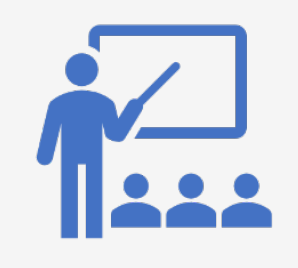

\section{Metodología}

Para la inclusión de las tecnología emergentes en el aula se debe elegir un diseño instrumental.

La estrategia se divide en tres momentos con una tecnología diferente en cada caso. 


\section{Metodología}

\section{Primera técnica}

Uso del cuento Letras, incógnitas y demás pesadillas
Mi primer pre-texto de álgebra, presenta la historia de dos estudiantes adolescentes que se preguntan sobre situaciones cotidianas de la escuela y de su vida que las llevan a cuestionar qué es una variable, cómo se representa con símbolos, qué operaciones se realizan con estos símbolos y para qué sirve representar los problemas cotidianos con expresiones simbólicas y algebraicas. 


\section{Metodología}

Segunda técnica

La app Cálculo con realidad aumentada

(Cálculo RA)
Explorará el concepto de límite de una función real mediante una aplicación de realidad aumentada donde un skater (patinador) se balancea en un half pipe (plataforma de patinaje).

Se estudia el concepto de variación y su representación para llegar a la expresión $\Delta y / \Delta x$, luego continúa con la exploración del concepto de límite. 


\section{Metodología}

\section{Segunda técnica}

La secuencia inicia con el profesor que indicará las instrucciones para el uso de la app, contempla cinco actividades:

\section{Primera actividad}

El alumno leerá las indicaciones de la app para la primera exploración, el acercamiento del patinador que se balancea en la plataforma de patinaje desde $\left(\mathrm{x}_{0}, \mathrm{y}_{0}\right)$ hasta llegar a un punto determinado que se identifica por las coordenadas $\left(\mathrm{x}_{1}, \mathrm{y}_{1}\right)$. 


\section{Segunda actividad}

El alumno deslizará al patinador que se balancea en la plataforma desde el lado izquierdo hasta donde se detiene, y describirá el comportamiento cuando el patinador tiende al valor $(0,0)$ y cuando tiende a

$(6,36)$, para posteriormente identificar la expresión

\section{Segunda técnica}

\section{$\lim _{T} x \downarrow \rightarrow a \uparrow-f(x)$.}

\section{Tercera actividad}

El alumno deslizará al patinador que se balancea en la plataforma desde el lado derecho hasta donde se detiene, y describirá el comportamiento cuando el patinador tiende al valor $(6,36)$ y cuando tiende a

$(0,0)$, para posteriormente identificar la expresión

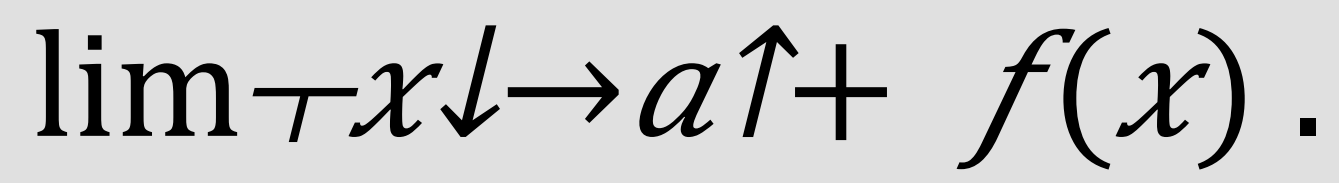




\section{Cuarta actividad}

El alumno deslizará al patinador que se balancea en la plataforma desde el lado derecho hasta el lado izquierdo, donde se detiene, y describirá el comportamiento cuando el patinador tiende al valor $(-9,36)$ y cuando tiende a $(0,0)$, para posteriormente identificar la

\section{Segunda técnica}

\section{expresión $\lim _{T} x \rightarrow a f(x)$}

\section{Quinta actividad}

El alumno deslizará al patinador que se balancea en la plataforma desde el lado derecho hasta el lado izquierdo, y describirá el comportamiento cuando el patinador se detiene en el valor $(-3,9)$.

Se le cuestionará sobre la expresión y su significado

\section{$\lim _{T} x \rightarrow-3 f(x+h)-f(x) / h$}

Finalmente, se le pregunta sobre el significado de la expresión y su interpretación con el patinador y la plataforma de patinaje.

\section{$\lim _{T} x \rightarrow 0 f(x+h)-f(x) / h$}




\section{Metodología}

\section{Tercera técnica}

\section{STI-m aplicación gradual} multidisciplinaria
La app muestra el tema de energía mediante una trayectoria de aprendizaje definida y recordatorios que ayuden al usuario a continuar explorando el contenido, solventar retos de aprendizaje y evidenciar su avance mediante niveles, como lo hace en un juego.

El realizar una actividad gradual multidisciplinaria mediante el STI$\mathrm{m}$ significa una aplicación que avanza por niveles a través de retos (gradual) y multidisciplinaria porque toma el tema de energía, pero lo aborda desde la perspectiva de la física, la química, la biología y las matemáticas. De manera que conforme avanza va profundizando en el concepto de energía y la manera de medir el cambio (variación). 


\section{Metodología}

\section{Tercera técnica}

EI STI-m incorpora a la arquitectura tradicional (módulo tutor, módulo dominio de conocimiento, módulo estudiante y módulo interfaz) un nuevo módulo diagnóstico, que identifica los atributos de un alumno para conocer donde comenzar su trayectoria de aprendizaje.

Luego determina el canal de aprendizaje preferido del alumno basado en el instrumento estándar Visual-Auditivo-Kinestésico (VAK) y el estado anímico.

Al final se integran pretest y postest para valorar el avance del estudiante. 


\section{Metodología}

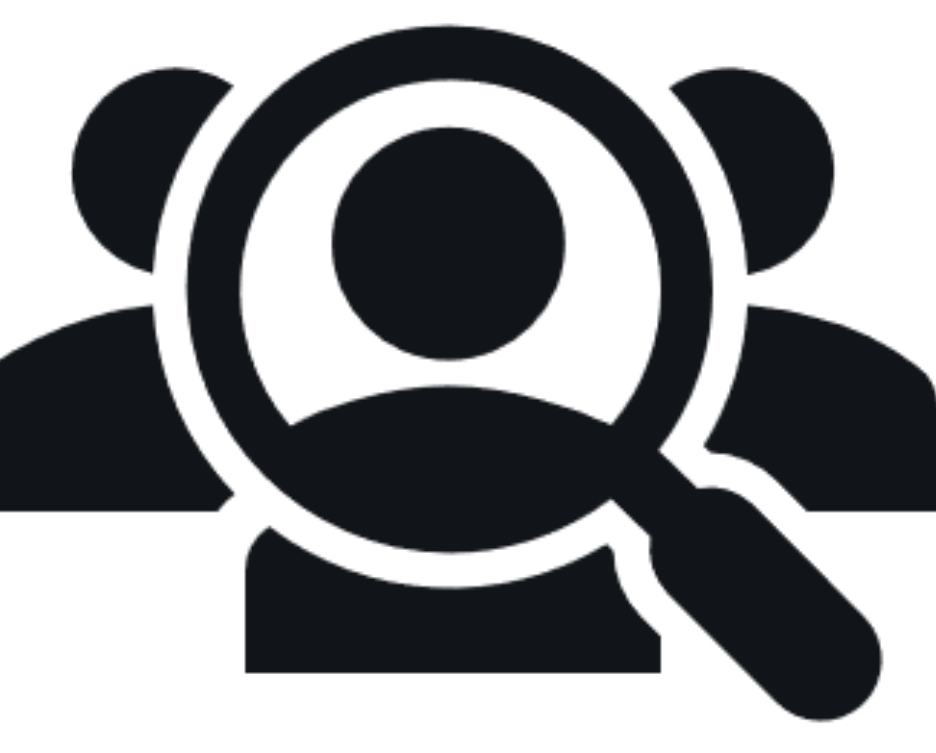

La población en los tres casos corresponde a 70 alumno de la licenciatura de Ingeniería en Computación, del Cen Universitario Valle de Chalco, organismo desconcentrad de la Universidad Autónoma del Estado de México. Los alumnos son de nuevo ingreso a la carreara, que han acreditado un examen de selección (EXANI I) y que se I encuesta sobre: el grado de aceptación de UAEMex Academy app y la app Cálculo RA, así como la utilidad d cuento Letras, incógnitas y demás pesadillas. Mi prime pre-texto de álgebra, bajo el enfoque del storytelling, pa explorar el uso de conceptos matemáticos sobre límites variación.

Se diseñaron instrumentos en cada caso y se analizaro mediante estadística descriptiva únicamente. 


\section{Resultados}

Para el caso del cuento Letras, incógnitas y demás pesadillas. Mi primer pre-texto de álgebra 


\section{Resultados}

Primero, se aplica un

cuestionario (pretest) para

explorar si los alumnos

identifican términos como

constante, variable,

parámetro, la forma de

representarlas y algunas

operaciones básicas que

se pueden realizar con

ellas.
- Después, se les pide a los estudiantes leer la historia de dos estudiantes adolescentes que se preguntan sobre situaciones cotidianas de la escuela y de su vida, en el lenguaje propio de la edad, y así llevan a cuestionar qué es una variable, cómo se representa con símbolos de la misma manera en que usan abreviaciones para mandar mensajes por celular mediante watts app y los emoticones de Facebook.
- A continuación, tratan de establecer operaciones que se realizan con estos símbolo y conocer para qué sirve representar los problemas cotidianos con expresiones simbólicas y algebraicas.

- Finalmente, se les pide a los estudiantes contestar un cuestionario que permita comparar sus respuestas iniciales del pretest con un postest, y anexar su opinión sobre la lectura y la utilidad de este recurso. 


\section{Resultados}

De los 70 alumnos de ingeniería, 50 indican que resulta adecuada la herramienta y es motivante para continuar explorando sobre álgebra, cambian su sentido de variable. Los restantes 20 consideran que la actividad es muy básica, aunque les resultó entretenido. 


\section{Resultados}

Para la exploración del concepto de límites permite estudia el comportamiento del patinador que se balancea en la plataforma de patinaje desde un punto inicial hasta llegar a un punto determinado.

Se le cuestiona al alumno sobre la diferencia entre $\left(x_{1}-x_{0}\right),\left(y_{1}-\right.$ $\left.\mathrm{y}_{0}\right)$, para diferentes valores de $\left(\mathrm{x}_{1}, \mathrm{y}_{1}\right.$, , para lograr llegar a la interpretación de $\Delta x, \Delta y$, finalmente a $\Delta y / \Delta x$ estudiando la función $y=f(x)=x^{2}$.

Luego mediante las tres actividades subsecuentes deslizará al patinador que se balancea en la plataforma desde el lado izquierdo hasta donde se detiene, lo mismo desde el lado derecho para llegar finalmente al límite sin importar el lado (izquierdo o derecho) por donde se acerca el patinador, ver figura 1. 


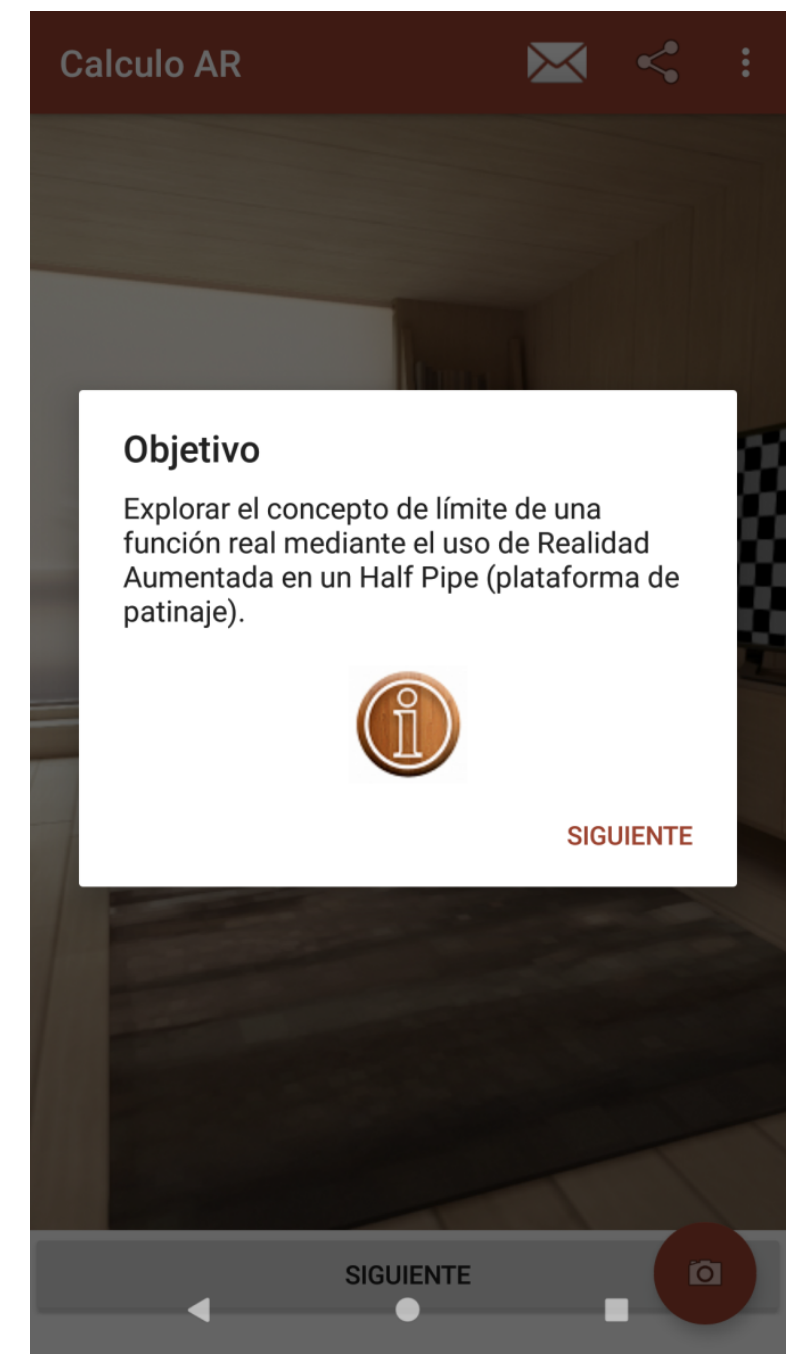

Figura 1. App Cálculo AR en actividad 1, 2 y 3. (Fuente: Elaboración propia)

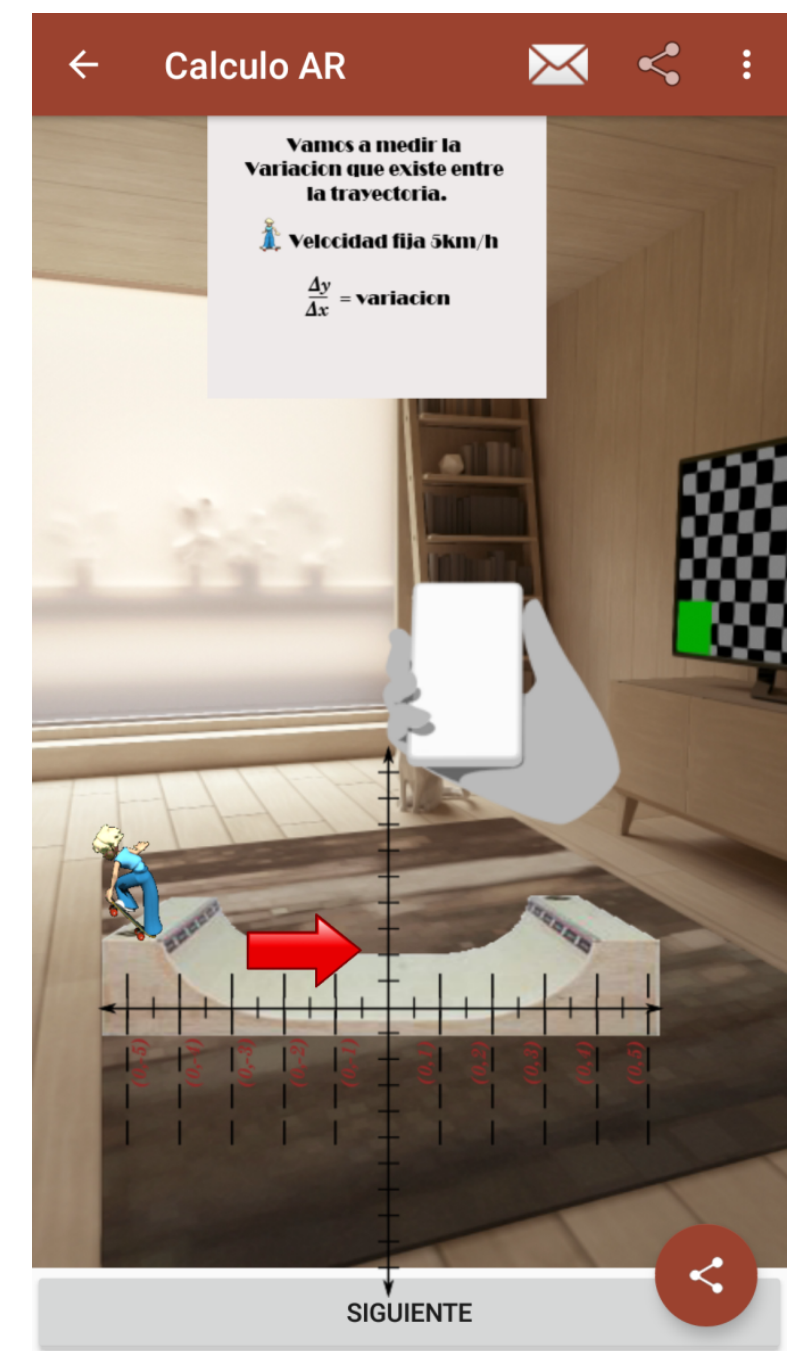

Figura 2. App Cálculo AR en actividad 4 y 5. (Fuente: Elaboración propia) 


\section{Resultados}

En las últimas dos actividades se describirá el comportamiento cuando el patinador se acerca a un valor determinado para generalizarlo y llegar a la expresión $\lim _{\boldsymbol{T}} \boldsymbol{x} \rightarrow \mathbf{0}$ $\boldsymbol{f}(\boldsymbol{x}+\boldsymbol{h})-\boldsymbol{f}(\boldsymbol{x}) / \boldsymbol{h}$ con un valor cualquiera.
De los 70 alumnos de ingeniería, 65 indican que resulta adecuada la herramienta y les permite pasar de un concepto de límite precario a una interpretación más acorde a la definición formal de límite. 


\section{Resultados}

\section{Sistema} Tutorial Inteligente móvil
La app dirige la actividad del alumno pasando de un tema básico a uno más complicado, con diferentes alternativas de presentación (visual, kinestésico, auditivo) y busca estrategias alternativas para que el alumno no se impaciente $y$ siga aprendiendo.

Además, presenta ejemplos a través de videos y simulaciones para que el estudiante desarrolle habilidades en la resolución de problemas por medio del pensamiento crítico. 


\section{Resultados}

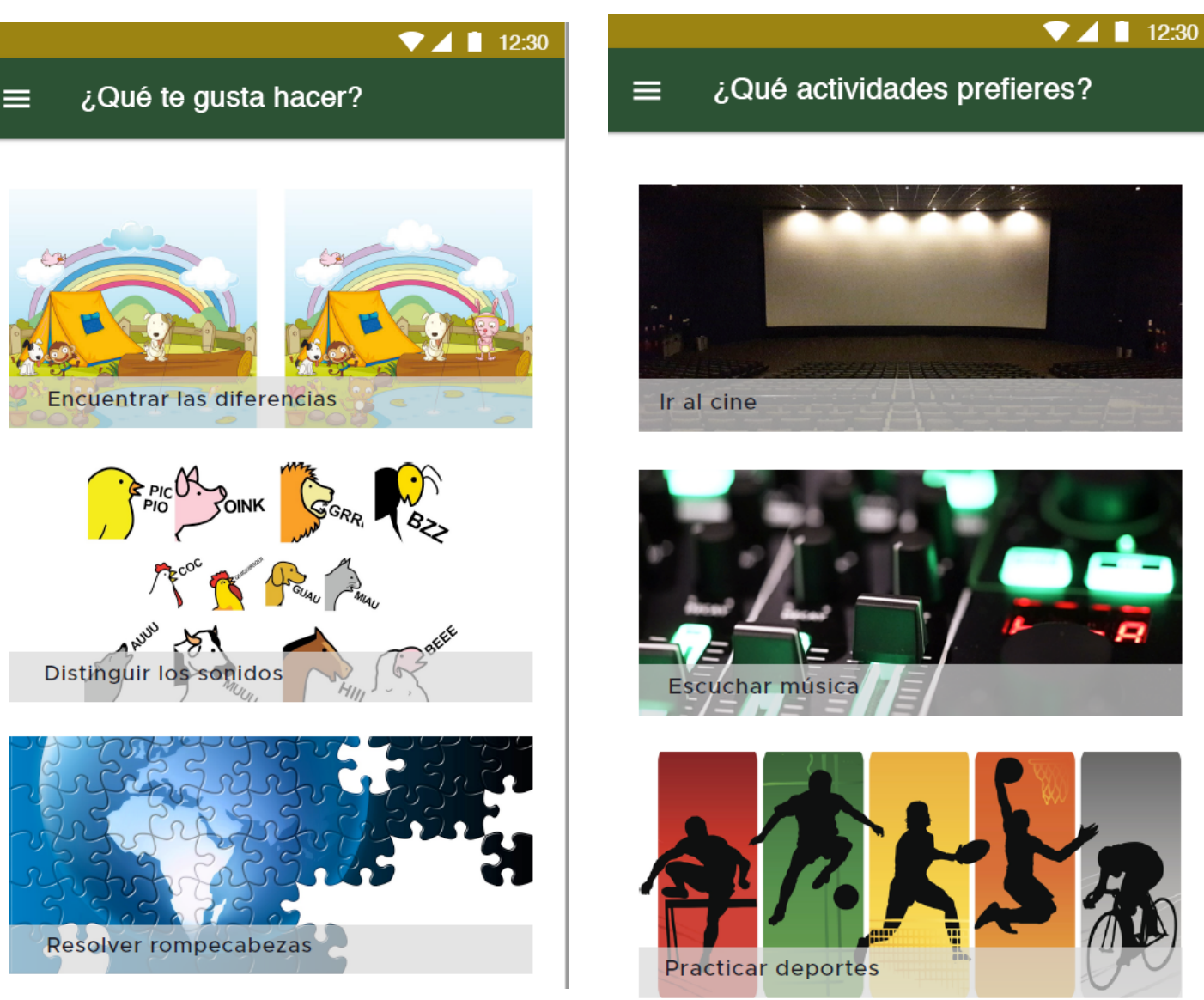

3. Ejemplo de Selección de canal de aprendizaje. (Fuente: Elaboración propia)
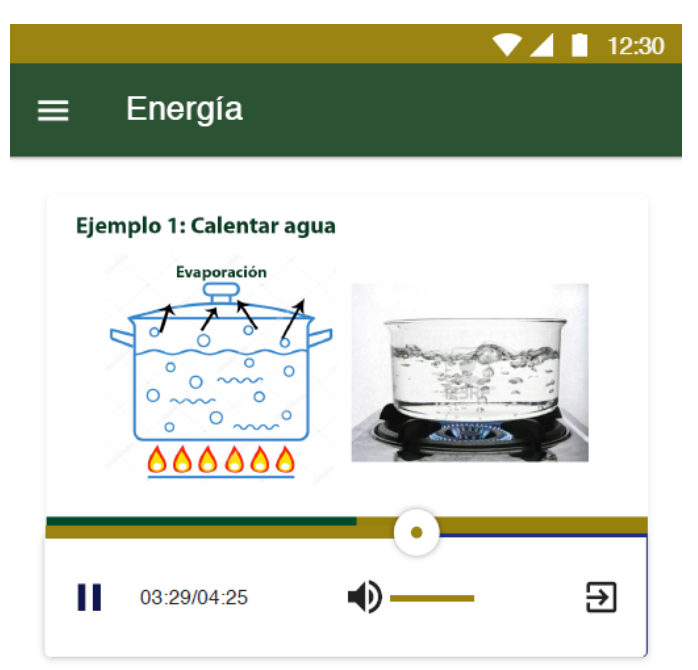

Concepto: Toda actividad requiere energía: mover una silla, para que un ser vivo se mueva, respire y se desarrolle, o para que las olas en una playa modelen el borde costero. Ningún proceso fisisco, quimico, biológico o geológico es posible sin ella. Pero ¿que
es la energín?

Por energía nos referimos comúnmente como la capacidad que
tienen los sistemas o cuerpos para modificar sus propiedades a lo tienen les sistemas o cuerpos
largo del tiempo, por ejemplo:

\section{Continuar}
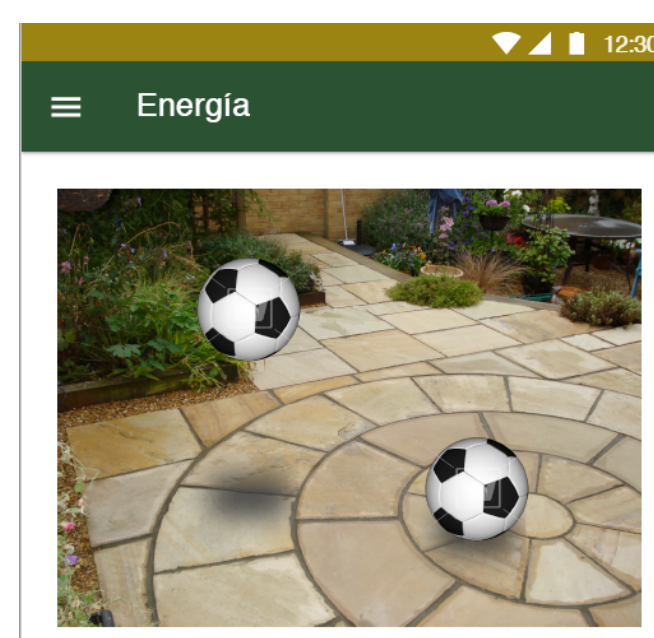

Concepto: Toda actividad requiere energia: mover una silla, calentar los alimentos o fabricar un utensilio. También es necesaria las olas en una playa modelen el bordde costero. Ningun proceso
fisico, químico, biologico o geológico es posible sin ella. Pero ¿qué es la energía?

Por energía nos referimos comúnmente como la capacidad que largo del tiempo, por ejemplos:

Continuar

Figura 4. Ejemplo de alternativas de presentación del mat para el tema de energía. (Fuente: Elaboración propia) 


\section{Resultados}

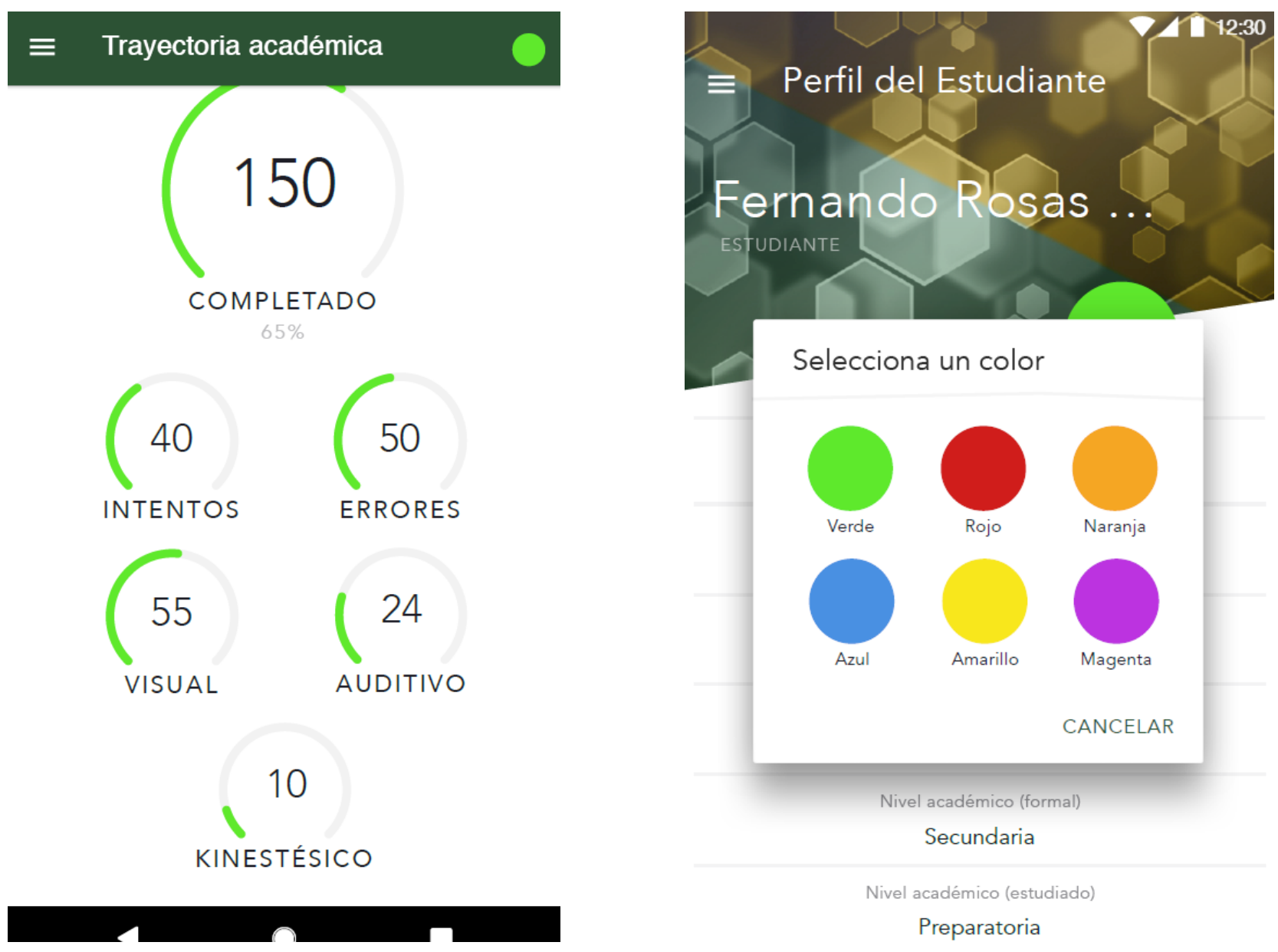

Figura 5. Ejemplo de resultados de seguimiento de evaluaciones y logros para el tema de energía. (Fuente: Elaboración propia) 


\title{
Conclusiones
}

\author{
Contar una historia \\ cotidiana y verídica \\ para que los alumnos \\ se vayan familiarizando \\ con términos como \\ variable, parámetro, \\ relación y la notación \\ apropiada desde el \\ álgebra, permite \\ enlazar la exploración \\ de conceptos \\ matemáticos básicos \\ hasta llegar a \\ conceptos más \\ profundos, lo que \\ cumple la finalidad del \\ cuento Letras, \\ incógnitas y demás \\ pesadillas.
}

La app Cálculo RA permite explorar el significado de uno de los conceptos más escabrosos del cálculo diferencial, el concepto de límite, la app gradualmente permite ir explorando el significado del concepto a través de la actividad del patinador, hasta llegar al concepto general de límite y el estudio de la variación con un significado para el alumno.
La aplicación gradual multidisciplinaria (UAEMex Academy app) en la modalidad de STI-m permite explorar la variación en el tema de energía, con la componente adicional de un acercamiento gradual y multidisciplinario, resultando un ejemplo de la estrategia de aprendizaje basado en problemas. 


\section{Conclusiones}

- Los tres ejemplos permiten ilustrar la propuesta de modelo de intervención didáctica basada en tecnologías emergentes que incorpora tecnología en el proceso enseñanza-aprendizaje tradicional, en el curso de cálculo diferencial con alumnos de primer semestre de Ingeniería en Computación del Centro Universitario UAEM Valle de Chalco.

- La propuesta se enmarca en el aprendizaje combinado (blended learning) mediante un diseño instrumental y didáctico fundamentado, se mide el uso de la tecnología y la incorporación de conceptos (cambio conceptual).

- Los resultados en 70 alumnos muestran un grado de aceptación de las app y el uso del cuento, los conceptos matemáticos explorados son utilizados en las diversas actividades incorporadas en las herramientas y validadas en el pretest generado un cambio conceptual. 